\title{
Active Control of Nano-dielectric-induced THz Quasi-BIC in Flexible Metasurfaces: A Platform for Modulation and Sensing
}

Thomas CaiWei Tan ${ }^{1,2}$, Yogesh Kumar Srivastava ${ }^{1,2}$, Rajour Tanyi Ako ${ }^{4}$,

\author{
Wenhao Wang 1,2,3, Madhu Bhaskaran ${ }^{4,5}$, Sharath Sriram 4,5, Ibraheem Al-Naib ${ }^{6}$,
} Eric Plum ${ }^{7}$ and Ranjan Singh ${ }^{1,2, *}$

${ }^{1}$ Division of Physics and Applied Physics, School of Physical and Mathematical Sciences, Nanyang Technological University, Singapore 637371, Singapore

${ }^{2}$ Centre for Disruptive Photonic Technologies, The Photonics Institute, Nanyang

Technological University, Singapore 637371, Singapore

${ }^{3}$ Institute of Fundamental and Frontier Sciences, University of Electronic Science and Technology of China, Chengdu 610054, China

${ }^{4}$ Functional Materials and Microsystems Research Group and the Micro Nano Research Facility, RMIT University, Melbourne, Australia

${ }^{5}$ ARC Centre of Excellence for Transformative Meta-Optical Systems, RMIT University, Melbourne, Australia

${ }^{6}$ Biomedical Engineering Department, College of Engineering, Imam Abdulrahman Bin Faisal University, Dammam, Saudi Arabia

${ }^{7}$ Centre for Photonic Metamaterials \& Optoelectronics Research Centre, Zepler Institute, University of Southampton, Southampton, SO17 1BJ, UK

*Email: ranjans@ntu.edu.sg

\begin{abstract}
A bound state in the continuum (BIC) is a non-radiating state of light embedded in the continuum of propagating modes providing drastic enhancement of the electromagnetic field and its localization at micro-nanoscale. However, access to such modes in the farfield requires symmetry breaking. Here, we demonstrate that a nanometric dielectric or semiconductor layer, 1000 times thinner than the resonant wavelength $(\lambda / 1000)$, induces a dynamically controllable quasi-bound state in the continuum (QBIC) with ultrahigh quality factor in a symmetric metallic metasurface at terahertz frequencies. Photoexcitation of nanostrips of germanium activates ultrafast switching of a QBIC
\end{abstract}


resonance with $200 \%$ transmission intensity modulation and complete recovery within 7 ps on a flexible substrate. The nanostrips also form microchannels that provide an opportunity for BIC-based refractive index sensing. We present an optimization model for (switchable) QBIC resonances of metamaterial arrays of planar symmetric resonators modified with any (active) dielectric for inverse metamaterial design that could serve as an enabling platform for active micro-nanophotonic devices.

\section{Introduction}

Engineering of passive and active narrowband resonances in small structures is of particular interest for miniaturization of resonant devices such as modulators, sensors, filters, and lasers. Hybrid metamaterial systems have been a strategy to incorporate the advantages of both subwavelength-scale metallic structure and tunable active control of semiconductors or phase change materials. Subwavelength metallic structures can be designed to support a broad range of resonances such as dipole, ${ }^{[1]} L C$-type, ${ }^{[1,2]}$ Fanotype $^{[3-7]}$ and EIT-like resonances. ${ }^{[8-11]}$ The active material then supplements and functionalizes the metamaterial by enabling resonance switching and modulation. For instance semiconductors such as silicon ( $\mathrm{Si})$, gallium arsenide (GaAs) and germanium (Ge) play a pivotal role in electronics and could also support active photonics. ${ }^{[12-17]}$ Active functionalities, such as switching and modulation, ${ }^{[18]}$ are required for dynamic control over signals, e.g. in communications, where miniaturization and speed are vital to address the growing bandwidth requirements and demand that we keep moving up in the frequency spectrum, eventually leading into the terahertz regime for the 
upcoming sixth generation $(6 \mathrm{G})$ telecommunications. ${ }^{[19]}$

Fano resonances are of particular interest as they can exhibit a high quality $(Q)$ factor and are suitable for applications that require high sensitivity and low loss. This includes lasing spasers, ${ }^{[20-23]}$ sensors ${ }^{[24-30]}$ and slow light devices. ${ }^{[13,16,31-33]}$. Fano resonances in both metallic and dielectric metasurfaces arise from small asymmetries that perturb a bound state in the continuum (BIC), allowing the perturbed state (quasi-BIC) possessing a finite linewidth to interfere with the incident plane wave. ${ }^{[14,34-42]}$ Theoretically, symmetry protected BICs are described as having an infinite lifetime and $Q$ factor. ${ }^{[42,43]}$ BICs have bound eigenmodes that exist above the photonic light line and are unobservable in the far-field. ${ }^{[38]}$ These modes are susceptible to external perturbations, which turn a BIC into a leaky Fano line-shape resonance with a finite lifetime ( $Q$ factor), which is also known as quasi-BIC (QBIC) resonance. ${ }^{[14,39]}$ The most common type of BIC is symmetry protected and the corresponding QBIC can be excited by perturbing a physical dimension of the resonator geometry to break its symmetry. This allows the resulting leaky resonance mode to couple to the free space and to be observed in the far-field. Such symmetry breaking is also a common strategy used to excite Fano resonances in metamaterials. A slight asymmetry between resonant components within a unit cell (e.g. two wires) of a periodic structure causes an imbalanced destructive interference between two radiation channels. ${ }^{[38,42]} \mathrm{A}$ balanced destructive interference would prevent leaky radiation to the far-field and corresponds to a BIC. 
In this work, we use nanometric thin semiconducting strips to perturb the BIC state by introducing a tiny out-of-plane nanoscale asymmetry in a terahertz resonant metamaterial based on symmetric metallic resonators, namely split-ring resonators (SRRs). ${ }^{[44]}$ Most previous demonstrations of symmetry broken metamaterials involve alterations in the lateral geometry of the resonant structures. ${ }^{[4,45]}$ The incorporation of the semiconducting material does not only result in a QBIC resonance but also allows modulation of the resonance. The combination of an active superstrate nanolayer on a metallic resonator enables the resonant unit cell to remain small compared to the wavelength, which is not possible in an all-dielectric configuration. This approach could potentially be used with any active dielectric material on any planar symmetric resonator array that supports a BIC mode. Here, we chose Ge to demonstrate ultrafast switching and modulation of QBIC resonances. The Ge strips also form microchannels, where an analyte could flow and enable refractive index sensing. We provide an optimization model for this system which can be used to design metasurfaces with a desired combination of QBIC resonance $Q$ factor and intensity.

\section{Results and Discussion}

Throughout this work, we will consider the properties of metamaterials illuminated at normal incidence (unless stated otherwise) by vertically $(y)$ polarized terahertz waves. We have shown in the supplementary information (Figure S1) by eigenmode and fullwave finite element analysis that under lossless conditions, a symmetric split-ring 
resonator possesses a high- $Q$ resonance. This high- $Q$ resonance has the characteristics of a BIC - a diverging $Q$ factor. Any symmetry-breaking perturbation of the BIC allows access to a narrow high- $Q$ mode. This strategy is commonly followed by creating an asymmetry in the lateral dimensions between the two constituent resonating arms by shifting one or both of the gaps slightly (e.g. micrometres in case of terahertz resonators).${ }^{[3]}$ In contrast, the method demonstrated here maintains the symmetry of the metallic resonator and incorporates a nanometric semiconducting layer on a portion of the unit cell. The chosen semiconducting material is germanium $(\mathrm{Ge})$ with a refractive index of $n_{\mathrm{Ge}}=4$. To disrupt the symmetry, a nanometric Ge strip of width $s=s_{L}$, is used to cover the left half of the unit cell as shown in Figure 1a(i). As the thickness, $h$, of the Ge layer increases, the $y z$ symmetry is broken and a resonance appears with a broadening linewidth and decreasing $Q$ factor. This can be seen in Figure 1a(ii), where the $Q$ factor that diverges at the symmetric point is proportional to $1 / h^{2}$ and $1 / \alpha^{2}$ with increasing thickness $h$ and asymmetry $\alpha$, respectively. The asymmetry parameter - introduced later - will quantify the asymmetry in terms of the key physical parameters that make the unit cell asymmetric and induce a QBIC resonance while preserving the symmetry of the metallic resonator. Figure $1 \mathrm{~b}$ highlights the dual$\mathrm{BIC} /$ symmetric nature of our system which is controlled by the non-metallic strip. The Ge strip of width $s=s_{L}+s_{R}$ is used to cover part of the left (L) half of the unit cell or all of the left half and part of the right $(\mathrm{R})$ half of the unit cell, as shown in Figure 1b. Starting from the centre of the unit cell, as the Ge strip is extended to cover the unit cell's left half, the unit cell becomes increasingly asymmetric and the QBIC emerges 
and redshifts in the transmission spectrum, from the BIC 1 frequency at $0.754 \mathrm{THz}$ to $0.66 \mathrm{THz}$ when the left half of the unit cell is fully covered. Further widening the strip into the right half results in a significant redshift as Ge coverage of the resonator's capacitive gap causes a capacitance increase and thus a resonance frequency decrease $\left(\omega_{0} \approx 1 / \sqrt{L C}\right)$. The resonance frequency continues to redshift until the unit cell is completely covered with Ge, which restores the symmetry of the structure, causing the QBIC to recover to BIC 2 at $0.61 \mathrm{THz}$. Thus, the cases of complete absence of the strip and complete strip coverage (and indeed any symmetric coverage, $s_{L}=s_{R}$ ) yield a BIC state. The QBIC resonance narrows and vanishes as these BIC states are approached. See Supplementary Figure S2 for the strip-width-dependent $Q$ factor of the QBIC resonance. Figure 1c shows the transmission amplitude spectra for (i, iii) the two BICs and (ii) the QBIC resonance of a half-covered system alongside their respective cross-sectional electric field at (i) $0.754 \mathrm{THz}$, (ii) $0.66 \mathrm{THz}$ and (iii) $0.61 \mathrm{THz}$. At BIC conditions, where the system is fully symmetric - fully (i) uncovered or (iii) covered with $\mathrm{Ge}$ - only a dipole resonance can be observed, and the electric field distribution has the same symmetry as the unit cell. An example of a symmetry broken BIC is shown in (ii), where the unit cell is half-covered with Ge (Half-Ge) and a sharp narrow resonance associated with an asymmetric field distribution appears at a lower frequency than the dipole resonance. We will use this narrow QBIC mode shown in (ii) to demonstrate switching and sensing in this paper. The addition of the semiconducting strip, which breaks the $y z$-plane mirror symmetry through the centre of the resonators, enables different excitation of the left and right resonator arms by normally incident $y$ - 
polarized THz waves. This can be seen in Figure 1c(ii), which shows the electric field $E_{z}$ on an $x z$ unit-cell cross-section of the metamaterial across one of the gaps, for illumination along $-z$. The presence of the Ge strip leads to the excitation of an asymmetric mode in the left (L) and right (R) resonator arms. The asymmetric excitation is associated with strong local fields as (approximately) destructive interference in the far-field traps energy in the metamaterial structure. However, the mode is not perfectly anti-symmetric, slightly stronger fields can be seen at the bottom of the Ge-covered half (L) of the unit cell. It is this deviation from the perfectly antisymmetric BIC mode that allows the asymmetric QBIC mode to leak and couple to the continuum as far-field radiation. This slight asymmetry of the excitation can also be interpreted as a resonance frequency mismatch of the two resonating arms, where the resonance frequency $\omega_{0}$ of the Ge-covered resonator arm is lowered. The symmetric local fields, plotted on the same scale, excited in the symmetric structure without the Ge strips are substantially weaker [Fig. 1c(i)]. The symmetric mode does not change much throughout the whole feature of the broad resonance, and constructive interference in the far-field implies that it is strongly coupled to the far-field and cannot trap much energy in the metamaterial device. The perfectly anti-symmetric BIC mode of the symmetric structures cannot be excited.

\footnotetext{
Device design and optimisation: $\mathrm{Ge}$ has an indirect bandgap of $0.66 \mathrm{eV}$ and a larger intrinsic carrier concentration and higher carrier mobility than silicon. Its ultrafast carrier dynamics and high absorption coefficient have also been appealing for photonic
} 
devices such as photodetectors or solar cells. ${ }^{[46,47]}$ As such, Ge is a suitable photoactive material for modulating or switching metamaterial resonances with light, removing the need for electrical contacts. The cyclic olefin copolymer (COC) substrate not only supplements the functionality by giving the device flexibility as shown in Figure 2a(i) but also minimizes the loss by having excellent electromagnetic characteristics (refractive index $n=1.52$ and loss tangent of $\tan \delta \sim 0.0006)^{[48,49]}$ at terahertz frequencies. Moreover, its refractive index is low enough to avoid lattice modes near the QBIC frequency. ${ }^{[50]}$ Images of a fabricated metamaterial array and its SRR unit cell are shown in Figure 2a(ii) and the inset reveals the dimensions of $95 \mu \mathrm{m}$ period, $80 \mu \mathrm{m}$ edge length, $6 \mu \mathrm{m}$ resonator line width and $4 \mu \mathrm{m}$ split gap. Gold is used for the SRRs and the COC substrate is $50 \mu \mathrm{m}$ thick in all cases. The Ge strip of this sample has a width $s=s_{L}=47.5 \mu \mathrm{m}$. Fabrication details are given in the experimental section. A QBIC that arises from breaking the symmetry of the unit cell with a dielectric strip can be optimized by adjusting the geometrical parameters of the dielectric strip, without changing the geometry of the metallic resonator. This concept allows enhancement in the functionality of any existing planar symmetric resonator array, that supports a BIC mode, with any available dielectric. This is illustrated in Figure 2(b-d), which shows the dependence of the SRR metamaterial's QBIC resonance on the strip's (b) width, (c) thickness and (d) material permittivity $\varepsilon$ (or refractive index $n=\sqrt{\varepsilon}$ for nonmagnetic materials). Simulated and measured transmission amplitude spectra of the metaphotonic device with a Ge thickness of $h=500 \mathrm{~nm}$ show that the QBIC resonance red-shifts $(0.7$ to $0.66 \mathrm{THz})$ and increases in amplitude as the strip width $s$ increases 
from 16 to $47.5 \mu \mathrm{m}$ (Figure 2b), where the $47.5-\mu \mathrm{m}$-wide (half-Ge) strip covers half of the unit cell. In contrast, the amplitude of the dipole resonance remains unchanged. The half-Ge strip structure was chosen for in-depth experimental characterization as it provides a resonance with a large amplitude and suitable linewidth for our measurement setup with a spectral resolution of about $30 \mathrm{GHz}$. Similarly, as the thickness of the halfGe strip is varied from 200 to $700 \mathrm{~nm}$ (Figure 2c), the QBIC resonance also redshifts (0.71 to $0.63 \mathrm{THz})$ and its amplitude also increases while the dipole resonance's amplitude remains unchanged. Using a thinner Ge layer, smaller than the penetration depth of $200 \mathrm{~nm}$ for a pump beam of $800 \mathrm{~nm}$ wavelength, ${ }^{[51,52]}$ could be advantageous for photoactive control as it requires less optical pump fluence to increase its photoconductivity ${ }^{[33]}$ however, thinner Ge also yields a weaker QBIC resonance. Considering this trade-off, a thickness of $500 \mathrm{~nm}$ was chosen. Measured and simulated resonances in Figures $2 b$ and $2 c$ match well in terms of spectral position. The contrast of experimental spectra is limited by the $30 \mathrm{GHz}$ spectral resolution of the terahertz time-domain spectroscopy (THz-TDS) setup. Figure 2d illustrates that any dielectric that provides some refractive index contrast can be used to obtain a QBIC resonance, for resonance optimization as well as sensing applications (later section). The larger the strip's permittivity, the thinner the strip can be for a measurable QBIC resonance, indicating that thinner strips made from a higher-permittivity semiconductor should be suitable for optical QBIC control at lower pump fluence. The dependence of the QBIC resonance on strip translation and additional results on strip width variation are shown in Supplementary Figures S3 and S4, respectively. 
In order to quantify and compare QBIC resonance characteristics, we fitted the transmittance spectra, $T(\omega)=|t(\omega)|^{2}$, as a Fano resonance $T_{F}$ (the QBIC resonance) on a Lorentzian background $T_{d}$ (the dipole resonance).

$$
\begin{aligned}
& T(\omega)=T_{d}+T_{F} \\
& T_{d}=T_{0}-I_{d} \frac{\left(\gamma_{d} / 2\right)^{2}}{\left(\omega-\omega_{d 0}\right)^{2}+\left(\gamma_{d} / 2\right)^{2}} \\
& T_{F}=-I_{F} \frac{(W+q)^{2}}{(1+q)^{2}\left(1+W^{2}\right)}, \quad W=\frac{\omega-\omega_{F 0}}{\gamma_{F} / 2}
\end{aligned}
$$

where the subscripts $d$ and $F$ correspond to dipole and Fano respectively, $\omega_{0}, \gamma$ and $I$ are frequency, FWHM and normalized intensity of the corresponding resonance, $T_{0}$ is the baseline shift of the whole spectrum, and $q$ is the asymmetry parameter that determines the QBIC resonance profile. Fitting examples are shown in Supplementary Figure S5.

The radiative losses of the QBIC resonance can be estimated by the $Q$ factor, $Q=$ $\omega_{F 0} / \gamma_{F}$. Another parameter of practical importance is the resonance intensity (depth) $I$, which can be extracted from the QBIC resonance peak and dip of the transmittance spectrum. As there is a trade-off between $Q$ factor and intensity of the QBIC resonance, ${ }^{[3]}$ we consider their product as a general figure of merit $(F o M), F o M=$ $Q \times I$, in order to find a desirable trade-off. The optimal trade-off will typically depend on additional application-specific considerations that cannot be captured in a general figure of merit. The $Q$ factor, intensity, and figure of merit of the QBIC resonance all depend on the strip-induced asymmetry. 
Here we describe this asymmetry $\alpha$ in a generalized form as the product of contributions associated with the strip area, its interaction with the metallic resonator, and its optical thickness.

$$
\begin{aligned}
& \alpha=\alpha_{\text {Area }} \times \alpha_{\mathrm{SRR}} \times \alpha_{\mathrm{OT}} \\
& \alpha_{\text {Area }}=\frac{2\left|A_{\mathrm{L}}-A_{\mathrm{R}}\right|}{A_{\text {unit cell }}} \\
& \alpha_{\mathrm{OT}}=\left|\frac{n_{\text {strip }}-n_{\mathrm{o}}}{n_{o}}\right| \frac{h}{1 \mu m} \\
& \alpha_{\mathrm{SRR}}=2-\left|\frac{4 d_{x, \text { asym }}}{P_{x}}\right|
\end{aligned}
$$

Such a definition of asymmetry, $\alpha$, is applicable to a unit cell which consists of two halves, "left" and "right", that are mirror images of each other. The symmetry that underpins a BIC corresponds to $\alpha=0$, which arises when the strip does not break the mirror symmetry of the unit cell, $\left(\alpha_{\mathrm{Area}} \times \alpha_{\mathrm{SRR}}\right)=0$, or when the strip is absent, $\alpha_{\mathrm{OT}}=0 . \alpha>0$ quantifies the asymmetry and leads to the emergence of the QBIC resonance.

Asymmetry results from any area of asymmetric strip coverage not affected by mirror symmetry from the two halves of the unit cell and it is quantified as $\alpha_{\text {Area }}$, the fraction of the unit cell that is covered asymmetrically. For a single strip oriented parallel to the symmetry axis ( $y$-axis), it is twice the difference between left and right strip areas, $A_{\mathrm{L}, \mathrm{R}}=s_{\mathrm{L}, \mathrm{R}} \times P_{y}$, normalized by the area of the unit cell, $A_{\text {unit cell }}$. As illustrated by Figure 3a, any part of a strip that is symmetric with respect to the symmetry axis does not contribute to area asymmetry (striped region) and the largest asymmetry of 1 corresponds to the case when the strip covers one half of the unit cell entirely, but not 
the other. Figures $1 \mathrm{c}(\mathrm{ii})$ and $2 \mathrm{~b}$ show that maximizing $\alpha_{\text {Area }}$ leads to a pronounced QBIC resonance.

The asymmetry caused by a strip that is placed asymmetrically on the unit cell also depends on the thickness $h$ of the strip and the difference between the refractive index of the strip $n_{\text {strip }}$ and the adjacent material covering the unit cell $n_{o}$ (air, or an analyte in case of sensing). This is illustrated in the bottom portion of Figure 3a. It is quantified by the optical thickness asymmetry, $\alpha_{\mathrm{OT}}$, which is the optical path length variation resulting from resonator illumination through the strip, divided by $1 \mu \mathrm{m}$ to arrive at a unitless quantity. (We anticipate that $\alpha_{\mathrm{OT}}$ could be generalized further by normalization to the wavelength instead, but testing this across a wide range of resonant wavelengths goes beyond the scope of this work.) Figure 2c shows that the QBIC resonance becomes more pronounced with increasing $\alpha_{\mathrm{OT}}$.

The area and optical thickness asymmetries described above are general and applicable to any dielectric strip placed on any symmetric, planar resonator. However, there is also a unit-cell-specific contribution to asymmetry, $\alpha_{\mathrm{SRR}}$, as some areas of a resonator can be more sensitive to the presence of the strip than others. As illustrated by Supplementary Figure S3, the SRR considered here is most sensitive to perturbations near the capacitive gap of the structure. Here we approximate this behaviour as linear, with $\alpha_{\mathrm{SRR}}$ increasing from zero when the asymmetric strip area is centred infinitesimally close to the edge of the unit cell to two when the asymmetric strip area is centred infinitesimally close to the middle of the capacitive gap. This is quantified in terms of the distance $d_{x \text {,asym }}$ from the symmetry axis to the middle of the asymmetric 
strip area, and the lattice period $P_{x}$, see Figure 3a. [If the strip covers the symmetry axis, $d_{x, \text { asym }}=\left(s_{\mathrm{L}}+s_{\mathrm{R}}\right) / 2$, otherwise $d_{x, \text { asym }}=d_{x}$, where $d_{x}$ is the distance of the centre of the strip from the symmetry axis.] As they both depend on the strip's placement within the unit cell, $\alpha_{\text {Area }}$ and $\alpha_{\mathrm{SRR}}$ are shown together in the top portion of Figure 3a.

Figures 3 shows how the (b) QBIC intensity, (c) $Q$ factor and (d) FoM depend on the generalised asymmetry parameter, $\alpha$. Various geometries were simulated to generate the figures, varying width, thickness, and position of the Ge strip to modify the asymmetry as explained in the figure and its caption. For all cases, the QBIC intensity and $Q$ factor follow an exponential trend as a function of asymmetry. With increasing asymmetry, the QBIC intensity increases exponentially, $I \approx 0.97-1.1 \exp (-0.46 \alpha)$, and the $Q$ factor decreases exponentially, $Q \approx 7+67 \exp (-0.84 \alpha)$, as shown in Figures $3 \mathrm{~b}$ and $3 \mathrm{c}$, respectively. Therefore, the $F o M$ can be fitted with the product of these exponential fits, and the optimal asymmetry can be obtained from the saddle point of Figure 3d. The optimal asymmetry of about $\alpha=1.5$ corresponds, for example, to the half-Ge SRR sample with $h=500 \mathrm{~nm}$. These results show how the asymmetry resulting from asymmetric placement of a dielectric strip on a symmetric unit cell can be quantified, and how this asymmetry is linked to the characteristics of the resulting QBIC resonance. By describing QBIC resonance intensity, $Q$ factor and $F o M$ as functions of all relevant physical characteristics of the strip (area, thickness, position, and refractive index), we provide a solution for the inverse design of QBIC metamaterials, from desired resonance characteristics to physical parameters of the unit 
cell geometry.

Ultrafast QBIC filter: The narrow QBIC resonance of the metal-semiconductor metamaterial structure could be exploited for spectral filtering, while the thin semiconductor layer offers an opportunity for active control of the device. Active control of the QBIC resonance is first investigated by CST simulations by changing the conductivity of Ge and then experimentally by optically pumping the device to excite charge carriers in the Ge strip. The increase of Ge conductivity due to photoexcitation of carriers in the conduction band changes the interaction between the incident $\mathrm{THz}$ waves and the metaphotonic device. As the Ge strip becomes more metallic, the interaction between the $\mathrm{THz}$ waves and the resonator, which is buried under Ge, is suppressed. Hence, the metamaterial changes from an SRR array to a strip grating coupled to half SRRs. Simulations of the transmission amplitude as a function of frequency and conductivity are presented in Supplementary Figure S6 and reveal three regimes of operation of the metaphotonic device: (a) bandstop, (b) intermediate switch off and (c) bandpass. However, switching to the bandpass mode requires an active material with higher photoconductivity.

The filtering and switching characteristics of the metaphotonic device have been measured by THz-TDS using an optical pump and a terahertz probe (OPTP) as described in the experimental section. The device is illuminated with a pulsed optical pump beam of $800 \mathrm{~nm}$ wavelength at different average power levels of 200, 600 and $1200 \mathrm{~mW}$ (pump fluences of 255, 764 and $1273 \mu \mathrm{J} / \mathrm{cm}^{2}$, respectively). As shown by Figure 4a, optical pumping yields substantial changes in the structure's transmission 
amplitude spectrum at the QBIC resonance (shaded region). The QBIC resonance becomes weaker with increasing pump fluence as predicted by the simulations shown in Supplementary Figure S6, i.e. the optical pump switches the narrow stopband of the filter off. We note that we limited the maximum experimental pump fluence to 1273 $\mu \mathrm{J} / \mathrm{cm}^{2}$ to avoid optical damage to the sample. Within the safe range of fluences, the increase in Ge conductivity is sufficient to suppress the QBIC resonance substantially, but the resonance does not vanish completely. While we are unable to experimentally demonstrate the simulated bandpass filter operation with this Ge-based device, the bandpass regime may be accessible with other materials such as semimetals. ${ }^{[54-56]}$ The spectra of Figure $4 \mathrm{a}$ were recorded with a pump-probe delay of 0 ps. Within experimental accuracy, Figure 4c(i), which shows the transmission amplitude modulation as a function of pump-probe delay, reveals that the maximum transmission modulation and Ge conductivity occur at this 0 ps delay, i.e., when the optical pump pulse interacts with the centre of the photoactive sample's surface while the THz probe pulse passes through the sample.

The change in transmission amplitude between the presence $\left(t_{\text {pump }}\right)$ and the absence $\left(t_{0}\right)$ of an optical pump is shown in Figure $4 \mathrm{~b}$. The maximum absolute transmission amplitude change obtained at the QBIC resonance is $t_{\text {pump }}-t_{0}=0.16$. This corresponds to amplitude modulation of $\frac{t_{\text {pump }}-t_{0}}{t_{0}} \times 100 \%=75 \%$ and intensity modulation of $\frac{t_{\text {pump }}^{2}-t_{0}^{2}}{t_{0}^{2}} \times 100 \%=200 \%$. At higher pump fluence, we also observed a large transmission change at higher frequencies, which is due to the broadening of the dipole mode and does not significantly affect the resonance amplitude and the switching 
capabilities of the device. The observed pump-induced transmission change is significant at the QBIC resonance frequency and small at the dipole resonance frequency, as highlighted by the red dashed lines in Figure 4b. Thus, we observe optical switching of a single resonance in the QBIC device.

The evolution of the transmission amplitude as a function of pump-probe delay carries information about the photoconductive carrier response in the metaphotonic device, i.e. the excitation and relaxation dynamics of the photo-carriers in the Ge strip, see Figure $4 \mathrm{c}(\mathrm{i})$. The pump pulse photoexcites carriers to the conduction band, resulting in a photoexcitation maximum at a delay of about $0 \mathrm{ps}$. The photoexcitation and relaxation are dependent on the pump fluence, as the photoexcitation peak of $\Delta t / t_{0}$ increases with increasing pump fluence, where $\Delta t=t_{\text {pump }}-t_{0}$ in the time domain. However, the photoexcitation peak should saturate exponentially at high pump fluence as the number of carriers that can be photoexcited to the conduction band is finite. ${ }^{[51]}$ The observed relaxation can be quantified through a single exponential fit and the time constant $\tau_{1}$ is presented in Table 1 for different pump fluences. ${ }^{[17,57,58]}$ We note that the metaphotonic device is at least as fast as the fitted time constant. The observation of comparable rise and decay times suggests that it could be much faster. A higher experimental temporal resolution would result from decreasing the angle of incidence of the pump beam $\left(\sim 10^{\circ}\right)$, which is not possible in our setup due to space constraints of the optical bench.

Table 1. Exponential time constants extracted from fitting Figure 4c(i) at different pump fluences. 
Figure $4 \mathrm{c}(\mathrm{ii})$ shows the temporal evolution of the QBIC resonance at different timestamps for a fixed pump fluence of $764 \mu \mathrm{J} / \mathrm{cm}^{2}$. At peak photoexcitation, the conductivity and the resulting transmission modulation at the QBIC resonance are the largest. As the photoexcited carriers relax, the Ge conductivity reduces and the QBIC resonance recovers, as shown in Figure $4 \mathrm{c}$ (ii) from 0 to $7 \mathrm{ps}$. This demonstrates ultrafast switching of the QBIC resonance, which recovers within 7 ps. Our results also demonstrate how either pump-probe delay or pump fluence can be used to modulate a QBIC resonance.

Microchannel QBIC refractive index sensor: Recently, I. Al-Naib ${ }^{[44]}$ reported refractive index sensing based on breaking the symmetry of an SRR through an analyte layer. Inspired by his work, our BIC-based refractive index sensor design uses a $\mathrm{Ge}$ layer on half of the unit cell to form a microchannel for an analyte to flow over the uncovered half, as shown in Figure 5a. Beyond liquid analytes, we note that unknown solids could also be characterized by deposition in the microchannels. In such devices, the optical path length difference for wave propagation through the analyte and the material covering the other half of the unit cell contributes to the symmetry breaking of the BIC and the spectral shift of the resulting QBIC. The larger the refractive index difference $(\Delta n)$ and the layer thickness $(h)$, the greater the optical path length difference $\Delta n \cdot h$ and the associated symmetry breaking and QBIC resonance shift away from the 
BIC resonance of the symmetric structure. Having the strip already in place improves and ease the fabrication for device integration. The choice of the strip material enables device optimization for operation in a desired refractive index range, where the QBIC is narrow. Figures $5 \mathrm{~b}$ and $5 \mathrm{c}$ illustrate the working principle of the device by showing how the transmission amplitude spectrum depends on the analyte's refractive index. The thickness of the superstrate (Ge and analyte) is kept constant at $h=500 \mathrm{~nm}$. We observe a collapse of the QBIC's linewidth as it approaches the BIC. Since the refractive index of $\mathrm{Ge}$ is 4 , this happens when the analyte's refractive index $n$ approaches 4 from either side. The horizontal white dashed line in Figure $5 \mathrm{~b}$ marks the eigenfrequency of the $\mathrm{BIC}(0.607 \mathrm{THz})$ calculated in COMSOL when the metamaterial is symmetric and completely covered with Ge, which matches well with the vanishing of the resonance in the transmission amplitude simulation in CST Studio Suite. We can quantify the sensitivity of the sensor as the resonance shift per refractive index unit of the analyte as shown in the inset of Figure 5c. The sensitivity obtained with a 500-nmthick superstrate is $\Delta f / \Delta n=-24.5 \mathrm{GHz} / \mathrm{RIU}$. The sensitivity in terms of wavelength is $\frac{d \lambda}{d n}=-\frac{c}{f_{0}^{2}} \frac{d f}{d n}=1.7 \times 10^{4} \mathrm{~nm} / \mathrm{RIU}$, where $c$ is the speed of light and $f_{0}=0.66 \mathrm{THz}$ is the QBIC resonance frequency without analyte. Our calculations reveal a moderate sensitivity reduction to $-19.5 \mathrm{GHz} / \mathrm{RIU}$ as the superstrate thickness is reduced to 300 $\mathrm{nm}$. This shows the versatility of the metaphotonic device design as a refractive index sensor for analyte layers of nanoscale thickness. The freedom to choose the superstrate material that forms the microchannels allows the BIC and nearby high- $Q$ QBIC to be shifted depending on device, integration, resolution, and application requirements. 
In summary, we demonstrated a novel class of active hybrid metamaterial devices and their inverse design. We showed how asymmetric placement of a dielectric or semiconductor strip with a nanoscale thickness on a symmetric metallic resonator of substantially subwavelength size induces a quasi-bound state in the continuum resonance. Through photoexcitation of semiconductor strips, we realized a narrow-band terahertz filter/modulator with $200 \%$ intensity modulation and recovery time within 7 ps, exceeding modulation depth and switching speed of comparable silicon devices. ${ }^{[12]}$ Due to their high flexibility, our structures could be integrated into wearable devices. Our results indicate that the use of the area in between the strips as microchannels for an analyte would result in a high-sensitivity refractive index sensor. We identified the relationship of $Q$ factor and intensity of the QBIC resonance with the unit cell's asymmetry. We enable the inverse design of QBIC metamaterials by expressing this asymmetry in terms of all relevant physical characteristics of the semiconducting or dielectric strip. Our approach offers a possibility to functionalize any planar symmetric resonator array, that supports a BIC mode, to an active device with a high-quality factor. Thus, this work could lead to the functionalization of many existing metamaterial configurations, that are based on symmetric resonators, to achieve switchable high- $Q$ resonances for the development and miniaturization of filters, modulators, and sensors.

\section{Experimental Section}

Sample fabrication: The symmetric Au SRRs were realized on COC films as detailed in our previous work. ${ }^{[40]}$ Here, a second photolithography process is conducted 
(MLA150 Maskless Aligner, Heidelberg Instruments) to carefully align strip patterns relative to the SRR split gaps prior to Ge deposition. Ge is deposited at a rate of $0.5 \AA / \mathrm{s}$ (Univex 250), followed by a lift-off process, where a sacrificial poly(methyl methacrylate) (PMMA) layer is dissolved in acetone, leaving the Ge strip-on-SRR resonators.

Terahertz time-domain spectroscopy (THz-TDS): The measurements are performed using ZnTe THz-TDS and the transmission amplitude is calculated by $t(\omega)=$ $\left|E_{S}(\omega) / E_{r}(\omega)\right|$, where the components of the numerator and denominator are the Fourier transforms of the time-domain electric field transmitted by the sample $s$ and reference substrate $r$, respectively. The metamaterial samples are illuminated at normal incidence by a $y$-polarized terahertz beam. An ultrafast Ti:Sapphire amplifier (Coherent Astrella) laser with $800 \mathrm{~nm}$ wavelength, $1 \mathrm{kHz}$ repetition rate and $<100 \mathrm{fs}$ pulse width, is used to generate the terahertz radiation in the ZnTe THz-TDS. A Zurich Instruments MFLI is used for lock-in detection.

Optical pump terahertz probe (OPTP): The dynamics are measured using the same ZnTe THz-TDS with the OPTP configuration. The device is illuminated at an oblique angle of incidence $\sim 10^{\circ}$ with a pulsed optical pump beam of $800 \mathrm{~nm}$ wavelength, $1 \mathrm{~cm}$ diameter, and $<100 \mathrm{fs}$ pulse width at different average power levels of 200, 600 and $1200 \mathrm{~mW}$ (pump fluences of 255,764 and $1273 \mu \mathrm{J} / \mathrm{cm}^{2}$, respectively). The width of the THz pulses is a few ps ( $\sim 2 \mathrm{ps})$ and the $\mathrm{THz}$ beam diameter is $7-8 \mathrm{~mm}$. The relative 
change in $\mathrm{THz}$ transmission is measured as $\Delta t / t_{0}$, where $\Delta t$ is the time domain change of THz transmission amplitude due to optical pumping and $t_{0}$ is the peak timedomain THz transmission amplitude without an optical pump. ${ }^{[59,60]} \Delta t$ depends on the optical pump probe time delay. Both $\Delta t$ and $t_{0}$ are measured using a multidemodulator lock-in (Zurich Instruments MFLI), where the delay line of $t_{0}$ is kept at peak $\mathrm{THz}$ signal and the delay line of the optical pump probe is varied (Figure 4c). Pump-fluence-dependent modulation is measured in the THz-TDS with the pump delay line at peak photoexcitation (Figures $4 \mathrm{a}$ and $4 \mathrm{~b}$ ).

\section{Supporting Information}

Supporting Information is available from the Wiley Online Library or from the author.

\section{Acknowledgments}

The authors acknowledge the funding support from the Singapore Ministry of Education (MOE) (Grant No. MOE2016-T3-1-006 and MOE2017-T2-1-110), National Research Foundation Singapore (Grant No. NRF-CRP23-2019-0005) and the UK's Engineering and Physical Sciences Research Council (Grants EP/M009122/1 and EP/T02643X/1). This

work was performed in part at the Micro Nano Research Facility at RMIT University in the Victorian Node of the Australian National Fabrication Facility (ANFF). Support from the Australian Research Council through the Centres of Excellence scheme (CE200100010) is acknowledged. The data from this paper is available from the University of Southampton ePrints research repository: https://doi.org/10.5258/SOTON/D1464. 


\section{References}

[1] N. Xu, R. Singh, W. Zhang, Applied Physics Letters 2016, 109, 021108.

[2] S. Linden, C. Enkrich, M. Wegener, J. Zhou, T. Koschny, C. M. Soukoulis, Science 2004, 306, 1351.

[3] L. Cong, M. Manjappa, N. Xu, I. Al-Naib, W. Zhang, R. Singh, Advanced Optical Materials 2015, 3, 1537.

[4] V. A. Fedotov, M. Rose, S. L. Prosvirnin, N. Papasimakis, N. I. Zheludev, Physical Review Letters 2007, 99, 147401.

[5] S. D. Jenkins, J. Ruostekoski, N. Papasimakis, S. Savo, N. I. Zheludev, Physical Review Letters 2017, 119, 053901.

[6] B. Luk'yanchuk, N. I. Zheludev, S. A. Maier, N. J. Halas, P. Nordlander, H. Giessen, C. T. Chong, Nature Materials 2010, 9, 707.

[7] T. C. W. Tan, E. Plum, R. Singh, Advanced Optical Materials 2020, n/a, 1901572.

[8] H. Guo, N. Liu, L. Fu, T. P. Meyrath, T. Zentgraf, H. Schweizer, H. Giessen, Opt. Express 2007, 15, 12095.

[9] B. Lahiri, S. G. McMeekin, R. M. D. L. Rue, N. P. Johnson, Applied Physics Letters 2011, 98, 153116.

[10]N. Liu, H. Giessen, Angewandte Chemie International Edition 2010, 49, 9838.

[11]T. C. Tan, Y. K. Srivastava, M. Manjappa, E. Plum, R. Singh, Applied Physics Letters 2018, 112, 201111.

[12]L. Wang, Y. Zhang, X. Guo, T. Chen, H. Liang, X. Hao, X. Hou, W. Kou, Y. Zhao, T. Zhou, S. Liang, Z. Yang, Nanomaterials 2019, 9, 965.

[13]J. Gu, R. Singh, X. Liu, X. Zhang, Y. Ma, S. Zhang, S. A. Maier, Z. Tian, A. K. Azad, H.-T. Chen, A. J. Taylor, J. Han, W. Zhang, Nature Communications 2012, 3, 1151 .

[14]S. Han, L. Cong, Y. K. Srivastava, B. Qiang, M. V. Rybin, A. Kumar, R. Jain, W. X. Lim, V. G. Achanta, S. S. Prabhu, Q. J. Wang, Y. S. Kivshar, R. Singh, Advanced Materials 2019, 31, 1901921.

[15] W. X. Lim, M. Manjappa, Y. K. Srivastava, L. Cong, A. Kumar, K. F. MacDonald, R. Singh, Advanced Materials 2018, 30.

[16]C. Lu, X. Hu, K. Shi, Q. Hu, R. Zhu, H. Yang, Q. Gong, Light: Science \&Amp; Applications 2015, 4, e302.

[17]P. Nagpal, V. I. Klimov, Nature Communications 2011, 2, 486.

[18]A. Kumar, A. Solanki, M. Manjappa, S. Ramesh, Y. K. Srivastava, P. Agarwal, T. C. Sum, R. Singh, Science Advances 2020, 6, eaax8821.

[19]Y. Yang, Y. Yamagami, X. Yu, P. Pitchappa, J. Webber, B. Zhang, M. Fujita, T. Nagatsuma, R. Singh, Nature Photonics 2020, 14, 446.

[20]N. I. Zheludev, S. L. Prosvirnin, N. Papasimakis, V. A. Fedotov, Nat Photon 2008, 2,351 .

[21] W. Zhou, M. Dridi, J. Y. Suh, C. H. Kim, D. T. Co, M. R. Wasielewski, G. C. Schatz, T. W. Odom, Nature Nanotechnology 2013, 8, 506.

[22] J. Y. Suh, C. H. Kim, W. Zhou, M. D. Huntington, D. T. Co, M. R. Wasielewski, T. W. Odom, Nano Letters 2012, 12, 5769.

[23]K. Tanaka, E. Plum, J. Y. Ou, T. Uchino, N. I. Zheludev, Physical Review Letters 
2010, 105, 227403.

[24]J. N. Anker, W. P. Hall, O. Lyandres, N. C. Shah, J. Zhao, R. P. Van Duyne, Nature Materials 2008, 7, 442.

[25]M. Islam, S. J. M. Rao, G. Kumar, B. P. Pal, D. Roy Chowdhury, Scientific Reports 2017, 7, 7355 .

[26]V. G. Kravets, F. Schedin, R. Jalil, L. Britnell, R. V. Gorbachev, D. Ansell, B. Thackray, K. S. Novoselov, A. K. Geim, A. V. Kabashin, A. N. Grigorenko, Nature Materials 2013, 12, 304.

[27]B. Lahiri, A. Z. Khokhar, R. M. De La Rue, S. G. McMeekin, N. P. Johnson, Opt. Express 2009, 17, 1107.

[28]R. Singh, W. Cao, I. Al-Naib, L. Cong, W. Withayachumnankul, W. Zhang, Applied Physics Letters 2014, 105, 171101.

[29]K. V. Sreekanth, Y. Alapan, M. ElKabbash, E. Ilker, M. Hinczewski, U. A. Gurkan, A. De Luca, G. Strangi, Nature Materials 2016, 15, 621.

[30]Y. K. Srivastava, L. Cong, R. Singh, Applied Physics Letters 2017, 111, 201101.

[31]Q. Bai, C. Liu, J. Chen, C. Cheng, M. Kang, H.-T. Wang, Journal of Applied Physics 2010, 107, 093104.

[32]M. Manjappa, S.-Y. Chiam, L. Cong, A. A. Bettiol, W. Zhang, R. Singh, Applied Physics Letters 2015, 106, 181101.

[33]A. Panahpour, Y. Silani, M. Farrokhian, A. V. Lavrinenko, H. Latifi, J. Opt. Soc. Am. B 2012, 29, 2297.

[34]D. R. Abujetas, N. van Hoof, S. ter Huurne, J. Gómez Rivas, J. A. Sánchez-Gil, Optica 2019, 6, 996.

[35]A. A. Bogdanov, K. L. Koshelev, P. V. Kapitanova, M. V. Rybin, S. A. Gladyshev, Z. F. Sadrieva, K. B. Samusev, Y. S. Kivshar, M. F. Limonov, Advanced Photonics 2019, 1(1), 016001.

[36]E. N. Bulgakov, A. F. Sadreev, Physical Review A 2015, 92, 023816.

[37]L. Cong, R. Singh, Advanced Optical Materials 2019, 7, 1900383.

[38]C. W. Hsu, B. Zhen, J. Lee, S.-L. Chua, S. G. Johnson, J. D. Joannopoulos, M. Soljačić, Nature 2013, 499, 188.

[39]K. Koshelev, S. Lepeshov, M. Liu, A. Bogdanov, Y. Kivshar, Physical Review Letters 2018, 121, 193903.

[40]Y. K. Srivastava, R. T. Ako, M. Gupta, M. Bhaskaran, S. Sriram, R. Singh, Applied Physics Letters 2019, 115, 151105.

[41]A. F. Sadreev, arXiv:2011.01221 [quant-ph] 2020.

[42]H. Friedrich, D. Wintgen, Physical Review A 1985, 32, 3231.

[43]J. von Neumann, E. P. Wigner, in The Collected Works of Eugene Paul Wigner: Part A: The Scientific Papers, (Ed: A. S. Wightman), Springer Berlin Heidelberg, Berlin, Heidelberg 1993, 291.

[44]I. Al-Naib, Journal of Infrared, Millimeter, and Terahertz Waves 2018, 39, 1.

[45]R. Singh, I. A. I. Al-Naib, M. Koch, W. Zhang, Opt. Express 2011, 19, 6312.

[46]Y. Kim, N. D. Lam, K. Kim, W.-K. Park, J. Lee, Scientific Reports 2017, 7, 42693.

[47]J. Michel, J. Liu, L. C. Kimerling, Nature Photonics 2010, 4, 527.

[48]K. Nielsen, H. K. Rasmussen, A. J. L. Adam, P. C. M. Planken, O. Bang, P. U. 
Jepsen, Opt. Express 2009, 17, 8592.

[49]F. Pavanello, F. Garet, M.-B. Kuppam, E. Peytavit, M. Vanwolleghem, F. Vaurette, J.-L. Coutaz, J.-F. Lampin, Applied Physics Letters 2013, 102, 111114.

[50]Z. F. Sadrieva, I. S. Sinev, K. L. Koshelev, A. Samusev, I. V. Iorsh, O. Takayama, R. Malureanu, A. A. Bogdanov, A. V. Lavrinenko, ACS Photonics 2017, 4, 723.

[51]T.-T. Yeh, H. Shirai, C.-M. Tu, T. Fuji, T. Kobayashi, C.-W. Luo, Scientific Reports 2017, 7, 40492.

[52]E. D. Palik, in Handbook of Optical Constants of Solids, (Ed: E. D. Palik), Academic Press, Burlington 1997, xvii.

[53]M. Manjappa, A. Solanki, A. Kumar, T. C. Sum, R. Singh, Advanced Materials 2019, 31, 1901455.

[54]P. Cheng, C. Zhang, Y. Liu, X. Yuan, F. Song, Q. Sun, P. Zhou, D. W. Zhang, F. Xiu, New Journal of Physics 2016, 18, 083003.

[55]W. Lu, J. Ling, F. Xiu, D. Sun, Physical Review B 2018, 98, 104310.

[56]Z. Dai, M. Manjappa, Y. Yang, T. C. W. Tan, B. Qiang, S. Han, L. J. Wong, F. Xiu, W. Liu, R. Singh, Advanced Functional Materials 2021, n/a, 2011011.

[57]W. Shockley, W. T. Read, Physical Review 1952, 87, 835.

[58]M. Zürch, H.-T. Chang, L. J. Borja, P. M. Kraus, S. K. Cushing, A. Gandman, C. J. Kaplan, M. H. Oh, J. S. Prell, D. Prendergast, C. D. Pemmaraju, D. M. Neumark, S. R. Leone, Nature Communications 2017, 8, 15734.

[59]Y. Shi, Q.-1. Zhou, C. Zhang, B. Jin, Applied Physics Letters 2008, 93, 121115.

[60]J. Hebling, M. C. Hoffmann, H. Y. Hwang, K.-L. Yeh, K. A. Nelson, Physical Review B 2010, 81, 035201. 
(a) (i) $s_{\mathrm{L}}=47.5 \mu \mathrm{m}, s_{\mathrm{R}}=0 \mu \mathrm{m}$

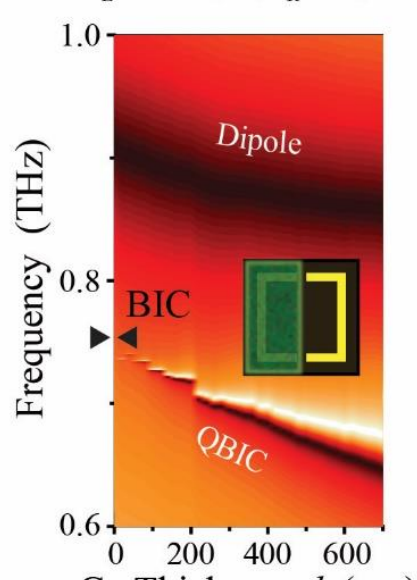

Ge Thickness, $h(\mathrm{~nm})$
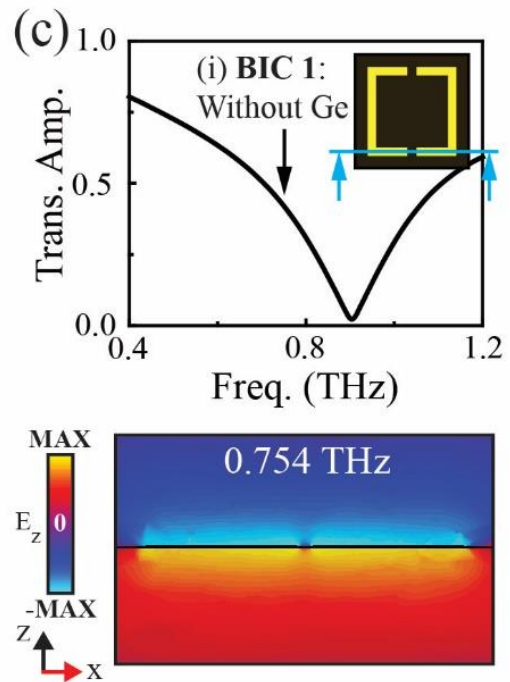

(b)
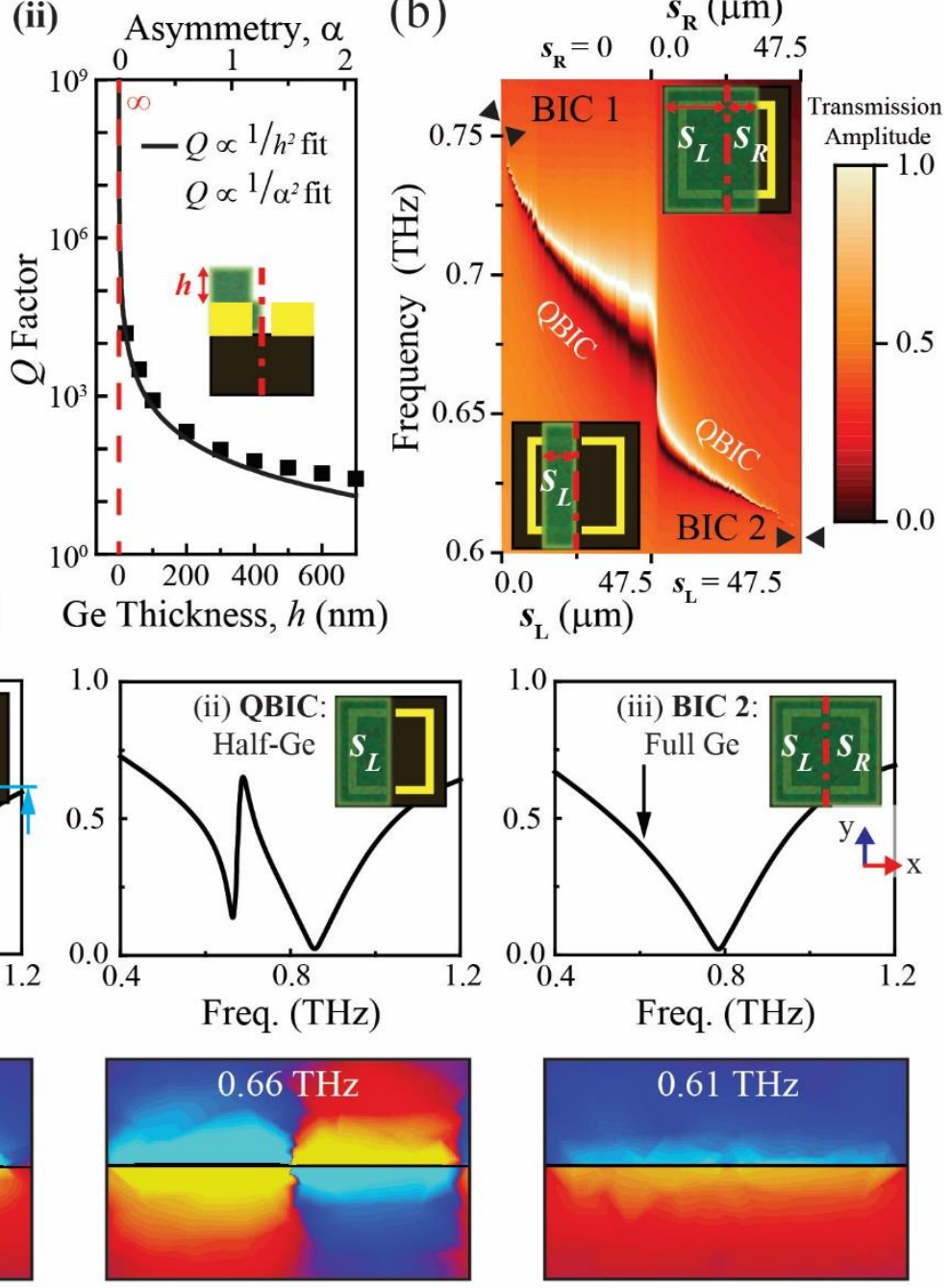

Figure 1: Dual bound states in the continuum of symmetric metamaterial covered by a nanodielectric strip. a) (i) Transmission amplitude spectra as a function of Ge strip thickness revealing a QBIC resonance. (ii) The $Q$ factor diverges as a function of Ge thickness and asymmetry with an inverse square trend that is typical for a BIC mode. b) Transmission amplitude spectra as a function of the width of a 500-nm-thick Ge strip covering part of the left $\left(s_{\mathrm{L}}\right)$ and right $\left(s_{\mathrm{R}}\right)$ halves of the $95 \mu \mathrm{m} \times 95 \mu \mathrm{m}$ unit cell. The two BICs correspond to the symmetric cases, e.g. absence of the $\mathrm{Ge}$ strip (BIC 1) and complete Ge coverage (BIC 2) of the unit cell. c) Transmission amplitude spectra and a cross-sectional electric field of (i) BIC 1: without Ge, (ii) QBIC: Half-Ge and (iii) BIC 2: Full Ge coverage with $500 \mathrm{~nm}$ thickness. The field maps show the $z$-component of the electric field across a unit cell (as indicated in blue) when the structure is illuminated by normally incident $y$ polarized electromagnetic waves at its (Q)BIC frequency. 

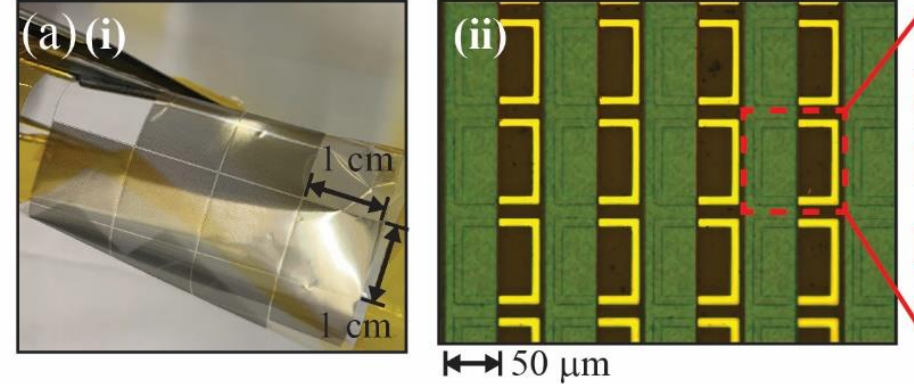

(b) $h=500 \mathrm{~nm}$

(c) half-Ge Strip

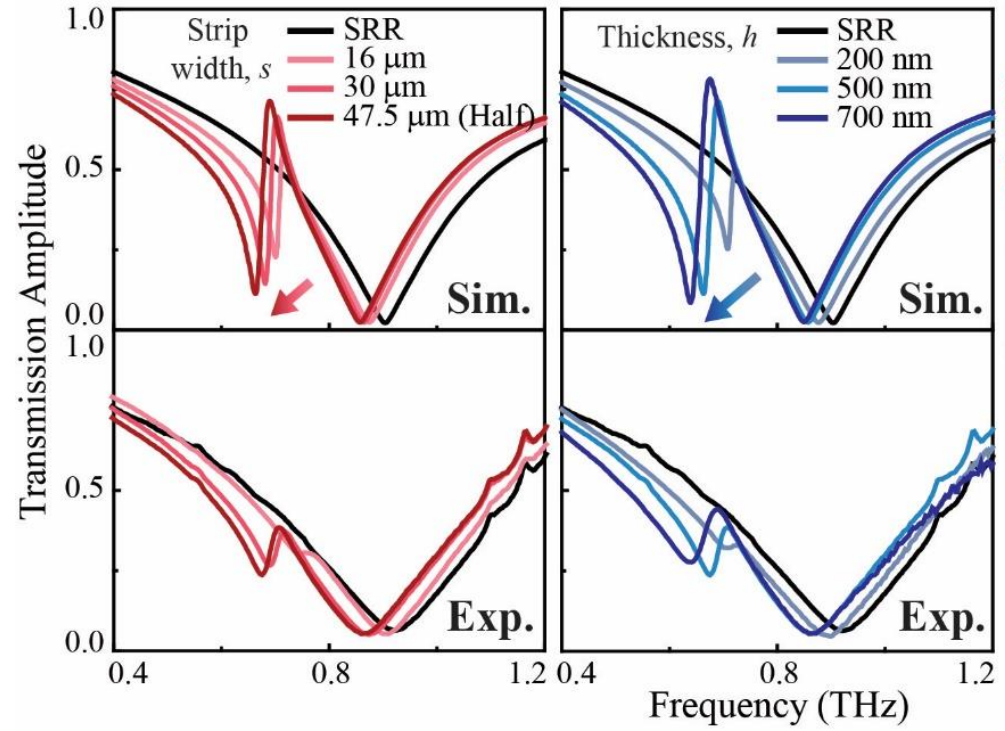

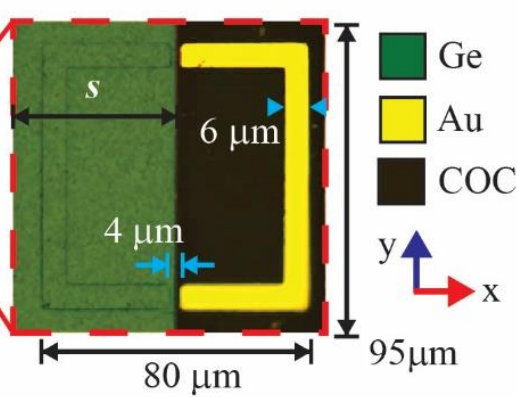

(d) $h=500 \mathrm{~nm}$, Half Strip

Figure 2: Metaphotonic device with a QBIC resonance determined by nano-dielectric strip design. a) (i) Flexible QBIC metaphotonic filter and sensor. (ii) Optical microscope image of the metaphotonic device with Ge strips of width $s=s_{L}=47.5 \mu \mathrm{m}$ and thickness $h=500 \mathrm{~nm}$ covering half of each unit cell as shown by the inset. (b-d) Simulated (top) and measured (bottom) transmission amplitude spectra for devices with (b) varying Ge strip widths from $s=16$ to $47.5 \mu \mathrm{m}$ for a fixed Ge thickness of $h=500 \mathrm{~nm}$, (c) Ge strips of varying thickness from $h=200$ to $700 \mathrm{~nm}$ with a fixed width of $s=47.5 \mu \mathrm{m}$ and (d) strips of materials with permittivity from $\varepsilon=1$ to 80 with fixed thickness $h=500 \mathrm{~nm}$ and fixed width $s=47.5 \mu \mathrm{m}$. 
ASYMMETRY, $\alpha=\alpha_{\text {Area }} \times \alpha_{\mathrm{SRR}} \times \alpha_{\mathrm{OT}}$

(a)STRIP WIDTH, $\boldsymbol{s}_{\mathrm{L}, \mathbf{R}} \&$ TRANSLATION $\boldsymbol{d}_{x}$

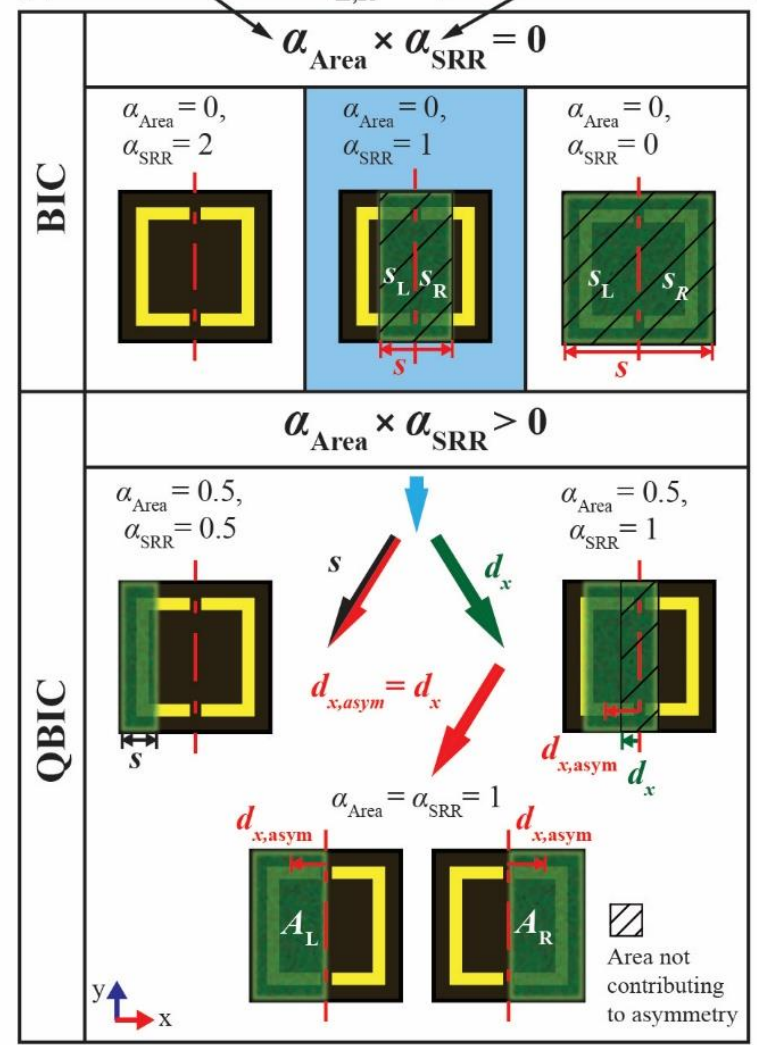

Ge thickness, $\boldsymbol{h}$
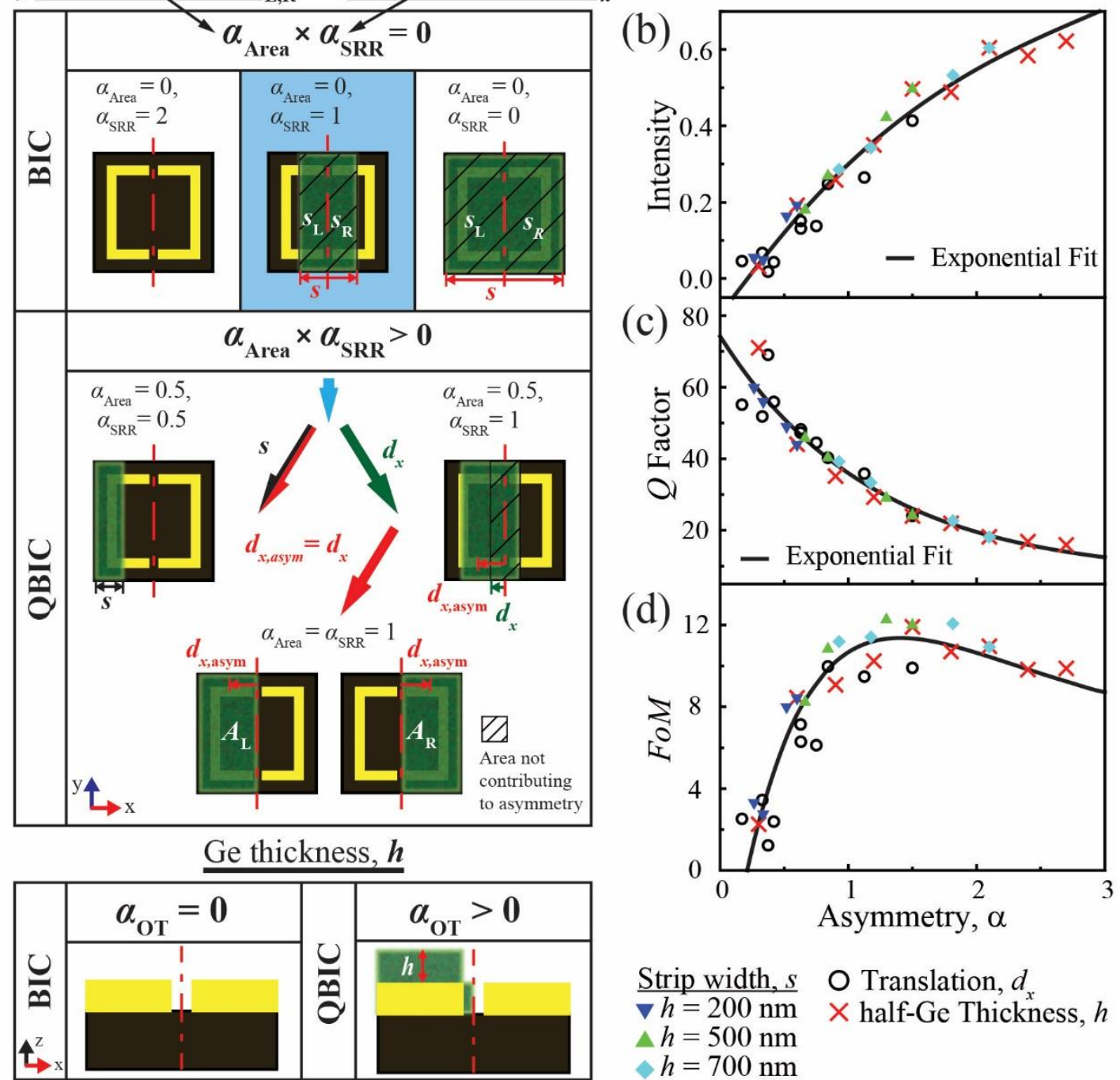

Strip width, $s$ O Translation, $d$ $\nabla h=200 \mathrm{~nm} \quad \times$ half-Ge Thickness, $h$ $h=500 \mathrm{~nm}$ $h=700 \mathrm{~nm}$

Figure 3: Quantifying asymmetry and its effect on the QBIC resonance intensity, $Q$ factor and Figure of Merit (FoM) based on simulations. (a) Asymmetry arising from the width, translation and thickness of a dielectric strip is described by asymmetric parameters; $\alpha_{\text {Area }}, \alpha_{\mathrm{SRR}}$ and $\alpha_{\mathrm{OT}}$. Their product is the general asymmetry parameter $\alpha$, where BIC conditions correspond to the absence of asymmetry $(\alpha=0)$ and QBIC conditions correspond to broken symmetry $(\alpha>0)$. (b - d) Increasing and decreasing exponential functions of asymmetry fit the (b) QBIC intensity and (c) $Q$ factor, and their product fits (d) FoM. Black circles represent the translation of 500-nm-thick Ge strips of either $s=16 \mu \mathrm{m}$ or $s=47.5 \mu \mathrm{m}$ width. Red crosses represent the thickness $h$ variation of half-Ge strips ( $s=47.5 \mu \mathrm{m}$ ) from 100 to $900 \mathrm{~nm}$. Strip width $s$ variation is represented by triangles and diamonds, where different symbols correspond to different Ge thicknesses as indicated and strips start from the centre and extend towards the left edge of the unit cell. 


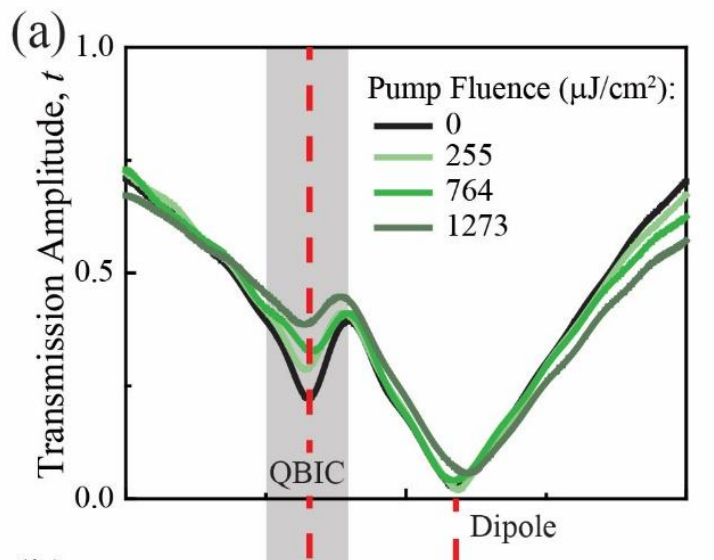

(c)
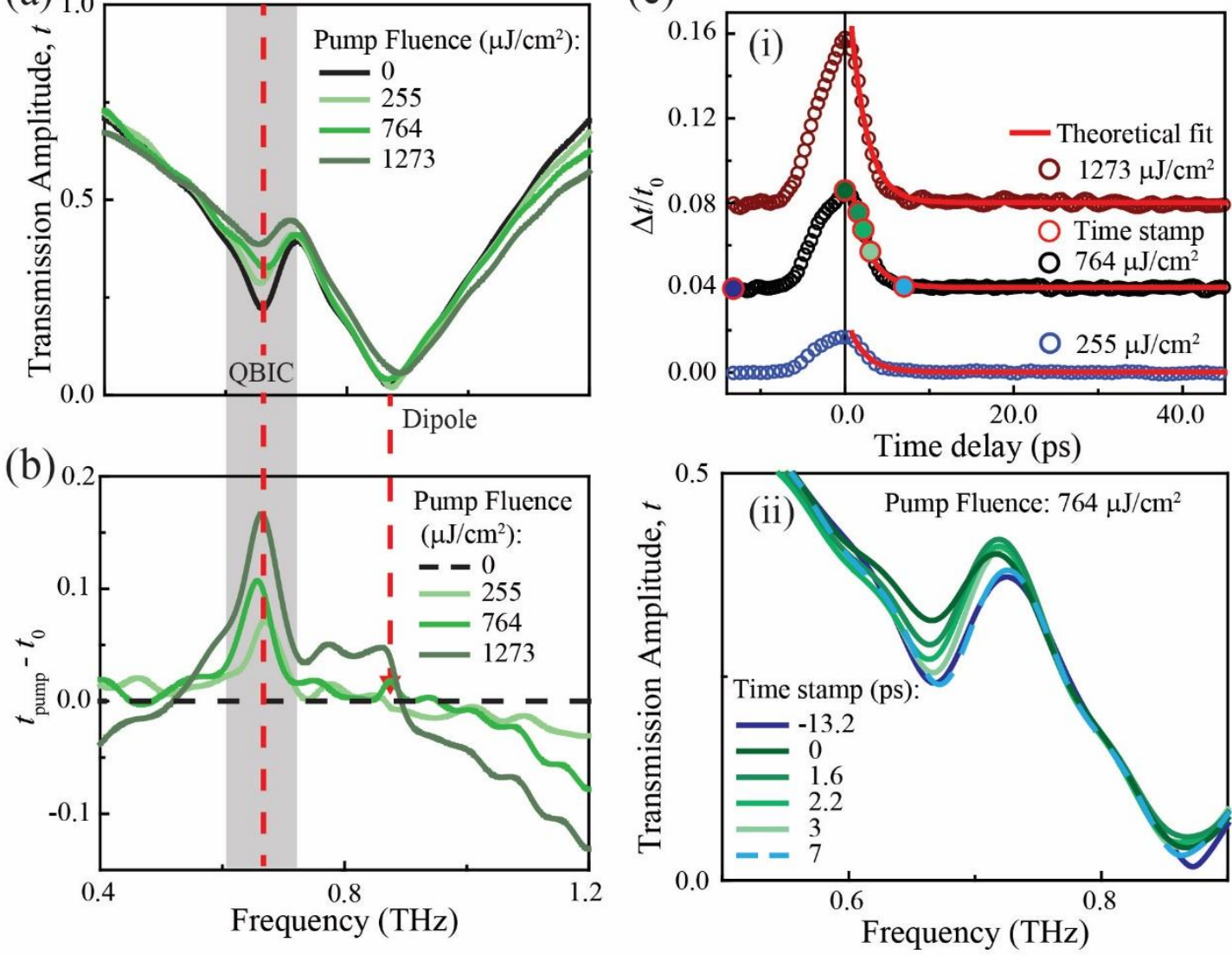

Figure 4: Optical control of the bandstop filter function of the metaphotonic device. a) Measured $\mathrm{THz}$ transmission amplitude spectra at different optical pump fluences. b) Absolute change of the transmission amplitude induced by the optical pump. The grey shaded area and red dashed line highlight the QBIC resonance while the red dashed arrow points to negligible absolute modulation at the dipole resonance. c) Measured optical pump-induced modulation. (i) Transmission amplitude change due to optical pump at the peak of the time-domain $\mathrm{THz}$ signal for different pump fluences. (ii) Transmission amplitude spectra measured at various time delays between the optical pump and $\mathrm{THz}$ probe at $764 \mu \mathrm{J} / \mathrm{cm}^{2}$ pump fluence, showing temporal modulation with complete recovery of the QBIC resonance within 7 ps. 


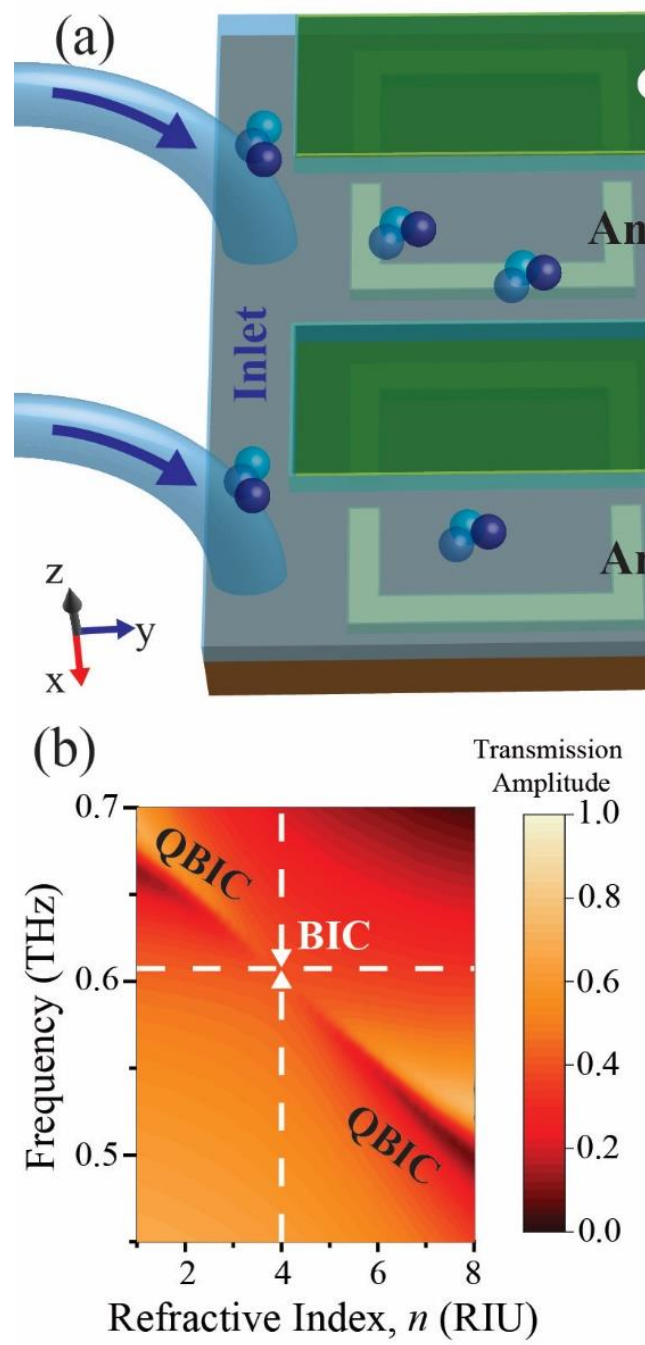

\section{Ge}
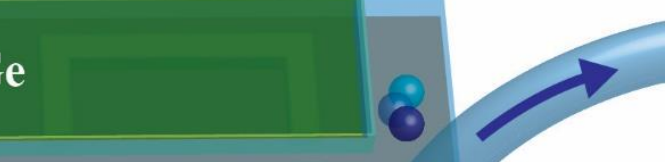

Ge

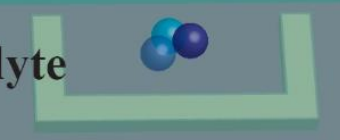

Ge
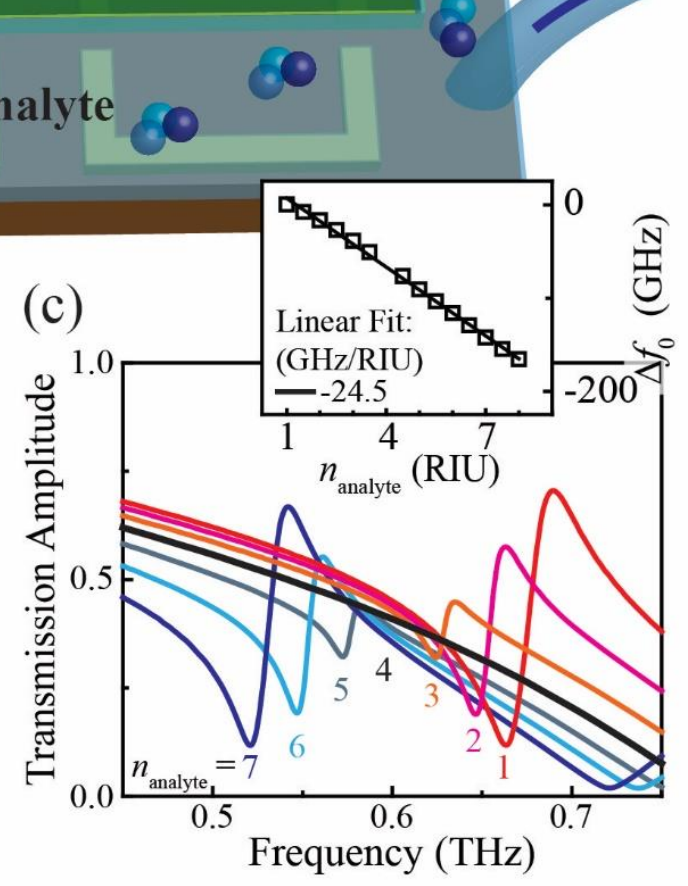

Figure 5: Terahertz refractive index BIC sensor. a) Illustration of the device with analyte flowing through the Ge strip microchannels. b) Simulated transmission amplitude spectra showing the collapse and revival of the BIC resonance mode with changing the refractive index of an analyte placed in microchannels separated by the Ge strips of the device of Fig. 2a. The refractive index of Ge is 4 (vertical dashed line), and the calculated BIC resonance (from COMSOL) is at $0.61 \mathrm{THz}$ (horizontal dashed line). c) Transmission amplitude spectra of the QBIC resonance for a superstrate thickness (Ge strip and analyte) of $h=500 \mathrm{~nm}$ and different refractive indices of the analyte. The inset shows the frequency spectral shift as a function of the analyte refractive index. The device sensitivity in terms of frequency shift per refractive index unit is obtained from the slope through a linear fit. 